DOI:10.30842/ielcp230690152529

\author{
D. Ivoška \\ (Institut für litauische Sprache, Vilnius, Litauen)
}

\title{
RUSSISCHE PERSONENNAMEN IN DEN FINANZIELLEN DOKUMENTEN DES DEUTSCHEN ORDENS
}

\author{
Д. Ивошка \\ (Институт литовского языка, Вильнюс, Литва)

\section{Русские личные имена в финансовых документах Немецкого Ордена}

Этнический состав средневековой Пруссии широко изучен, так как он очень хорошо отражен в документах, свидетельствующих о политике Немецкого ордена (лат. Ordo Teutonicus, нем. Deutscher Orden) по колонизации и заселении свободных территорий. Экономическая, сельскохозяйственная, социальная, религиозная и культурная системы, сформированные на дохристианских прусских землях, были преобразованы Немецким орденом. Смешение крупных этнических групп, т. е. пруссов, немцев и поляков, также оказало влияние на этот процесс. Позже, особенно в Восточной Пруссии, во многих социокультурных процессах участвовали литовцы, а в польской Померании стала подчёркиваться роль кашубов. Помимо указанных национальностей, в документах Немецкой орденской канцелярии зафиксированы русские, проживающие в Пруссии, но вопрос об этой этнической группе практически не получил внимания со стороны историков. Некоторые историки упоминают русских Пруссии в своих работах, но не анализируют, как эта этническая группа возникла на прусских территориях, не говоря уже об общественных отношениях русского населения, религиозных и культурных проблемах, а также о правах собственности в Пруссии. В связи с этим возникают следующие вопросы: почему факт проживания русских в Пруссии так мало изучен? Было ли русских, живущих на прусских землях, слишком мало и поэтому этот факт не получает должного внимания? А может просто не хватает данных для углублённого исследования? В данной статье анализируется фактический материал, собранный из одного из важнейших финансовых документов Немецкого ордена - Мариенбургской казначейской книги 1399-1409 г2. (нем. Das Marienburger Tresslerbuch der Jahre 1399-1409), представлены упомянутые в нем русские личные имена и связанные с ними данные.

Ключевые слова: русские личные имена, Немецкий Орден, тенденции именования. 
D. Ivoška

(Institute of the Lithuanian Language, Vilnius, Lithuania)

\section{Russian Personal Names in Financial Documents of the German Order}

The ethnic composition of medieval Prussia has been extensively studied, as it is very well reflected in the documents testifying to the German Order's policy of colonization and settling free territories. The economic, agricultural, social, religious and cultural systems formed in the pre-Christian Prussian lands were transformed by the Order. The mix of large ethnic groups, i.e. the Prussians, Germans and Poles, also had influence on this process. Later, especially in East Prussia, many sociocultural processes involved Lithuanians, while in the Polish Pomerania the role of Kashubians began to be emphasized. Besides the mentioned nationalities, documents of the German Order Chancellery recorded Russians living in Prussia, but the question of this ethnic group has received practically no attention from historians. Some historians mention Russians of Prussia in their works, but do not analyze how this ethnic group emerged in Prussian territories, not to mention Russian population's social relations, religious and cultural issues as well as property rights in Prussia. The question is, were there too few Russians living in Prussian lands that they have not received scholars' due attention, or is there simply a lack of data for the in-depth research? This article analyzes the factual material collected from one of the most important financial documents of the German Order - the Marienburg Treasurer's Book 1399-1409 (Ger. Das Marienburger Tresslerbuch der Jahre 1399-1409) — and presents the Russian personal names and related data mentioned in it.

Keywords: Russian personal names, German Order, naming tendency.

\section{Russenfrage in dem mittelalterlichen Prußenland.}

Die Bezeugungen über die von dem Deutschen Orden (im Weiteren - DO) nach der Eroberung aller einzelner prußischen Stämme Ende des 12. - Anfang des 13. Jhs. ${ }^{1}$ unternommene Besiedlungs- und Christianisierungspolitik des Landes ist in dessen vielen Dokumenten überliefert und von zahlreichen Gerichtsschreibern sowie Historikern häufig behandelt worden. In dem vorliegenden Beitrag wird der Schwerpunkt von Einwohnerherkunft des Prußenlandes Ende des 14. - Anfang des 15. Jhs. behandelt und grundsätzlich versucht werden, die Frage der Russen zu untersuchen, deswegen werden die Ereignisse und sie bezeugende Dokumente diplomatisch-politischen Charakters nicht angegangen.

\footnotetext{
${ }^{1}$ Zum Überblick über die Eroberungsgang der prußischen Stämme s. Ivoška (2019a: 7).
} 
Die allgemeine Kenntnis über die Bevölkerungszusammensetzung des dem DO unterworfenen Prußenlandes, besonders nach dem Vollenden maßgeblicher Aktionen der Siedlungstätigkeit besteht auf drei Nationalitäten: prußischer, deutscher und polnischer. Die Verhältnisse der Bewohner, die in einem riesigen Territorium, das sich zwischen den Mündungen der Weichsel und der Memel sowie zwischen dem Kulmerland, die Grenze Masowiens entlang bis an die Grenze mit Litauen, erstreckt hatte, hatten sich im Grunde genommen unter den ansässigen Prußen, Polen und den eingewanderten Deutschen entwickelt. Die Besiedlungsvorgänge sind auf Grund der Ordensdokumente ausführlich von Karl Kasiske (1934) behandelt. Seine Forschungen umfassen aber den westlichen Teil des Prußenlandes nicht, was Kurt Forstreuter (1937: 501) in seiner Buchbesprechung zum Kasiskes Werk bedauert hatte. Die durch den DO geführte Kolonialpolitik verursachte nicht nur die Völkerwanderung in dem Prußenland intern, sondern trug auch zu der Zuwanderung anderer Völker nach Prußenland bei. Im 14. - 15. Jh. werden als Einwohner des Prußenlandes neben Prußen, Polen und Deutschen auch Litauer, Kaschuben und Kuren erwähnt (Wenskus 1975: 419, 437). Eine gründliche und umfangreiche Übersicht über die ethnischen Verhältnisse und die Anteile verschiedener Nationalitäten gab auch Marian Biskup (1991: 6-16, 46-52) an. Mit dem Schwerpunkt der Zuwanderung in das mittelalterliche Prußenland hat sich auch Janusz Małłek (2003: 432440) befasst. Der Autor beschäftigt sich vorwiegend mit den deutschen, polnischen und litauischen Zuwanderern, nennt aber kurz auch die Vertreter anderer ethnischen Gruppen, die einen sehr kleinen Anteil der Bevölkerung in Prußenland ausmachten. Die Bevölkerungszusammensetzung im Preußisch Litauen hat Gertrud Mortensen (1927) behandelt. Alle angeführten Autoren weisen aber mit keiner kleinsten Andeutung auf die Russen in dem Prußenland, obwohl die Dokumente des DO ihre Anwesenheit im Lande bestätigen. Die Ursache dafür mag, vielleicht, laut Peter Erlen (1992: 71) die Tatsache sein, dass deren spätere Einwanderung ,,in der Blütezeit der mittelalterlichen Besiedlung eine unbedeutende Rolle gespielt hatte". Erlen lenkt trotzdem kurz die Aufmerksamkeit auf die ,nichtdeutschen und nichtprußischen Bevölkerungsgruppen“", unter denen er sich im Grunde genommen mit den Polen und Kaschuben beschäftigt, berührt aber kurz auch die Russen- und Litauerfrage. Mit einer kargen Anmerkung deutet auch Forstreuter (1937: 501) darauf, dass es unter nach 1410 zugewanderten Polen und Litauern auch Russen gab. Die Frage, woher sie zu der Zeit 
einwandern konnten, bleibt aber unbeantwortet. Die nahe Nachbarschaft mit dem von Litauern und Russen bewohnten Masowienwinkel, der sich zwischen dem prußischen Sudauen und Litauen ausgedehnt hatte ${ }^{2}$, sowie mit der jatwingischen Landschaft, mag auch die Grundlage zur leichten Migration aus wirtschaftlichen, kriegerischen u.a. Anlässen geschaffen haben. Eine weitere Tatsache, die die Wanderung von Russen aus Polen verursacht haben konnte, mag die seit dem 13. Jh. bei der Verleihung von dem Landbesitz durch polnische Herzöge angewandte ius Theotonicum, die später durch die Regelung Exceptis schismatis erweitert worden war, gewesen sein. Das Genießen von Vorteilen der deutschen erbrechtlichen Regelung wurde durch die Politik Kasimir des Großen, später auch durch die von Władysław Jagiełło in Polen sowie durch Wytowt in Litauen, den Anhängern des nicht-katholischen Glaubens ausgeschlossen (Janeczeck 1984: 527-529). Die in dem Ordensfolianten Nr. 105 (im Weiteren - OF 105) enthaltenen Handfesten des DO von dem Jahr 1402 zeugen aber dagegen von 3 Russen Demyan, Radewayn und Carpe, denen je 4 Huben Acker unter denselben Bedingungen, wie den einheimischen Prußen zu Angerburg $^{3}$ verliehen wurden (OF $\left.105119^{\mathrm{r}}\right)^{4}$.

Als Hauptquelle der vorliegenden Untersuchung wurde „Das Marienburger Tresslerbuch der Jahre 1399-1409“ (im Weiteren MTB) gewählt, weil es zahlreiche Namen russischer Herkunft enthält. Das im Jahr 1896 von dem Königsberger Staatsarchivrat Erich Joachim herausgegebene MTB ist die Druckauflage des handgeschriebenen, heute in dem Geheimen Staatsarchiv „Preußischer Kulturbesitz" aufbewahrten Ordensfolianten Nr. 140. Das MTB ist, laut dem Herausgerber, ,nichts andres als das Hauptbuch über Einnahme und Ausgabe der Ordensstaatskasse“. Etwas ihm Ähnliches habe sich weder aus den Zeiten vorher noch aus den späteren erhalten (MTB IV) ${ }^{5}$. Dieser Ordensfoliant ist vielleicht der einzige unter anderen, das Spätmittelalter umfassenden handschrift-

\footnotetext{
${ }^{2}$ Zofia Kowalska-Urbankowa (1987: 214-215).

${ }^{3}$ Angerburg, $30 \mathrm{~km}$ von Gerdauen (Blažienè 218: 118).

${ }^{4}$ Die in dem OF 105 gebuchten Namen der Russen wurden von Grasilda Blažienè in ihrem Beitrag ,Zu den russischen Personennamen in den Folianten des Deutschen Ordens" (2018: 118-122) behandelt. Es muss bemerkt werden, dass alle drei in dem OF 105 genannten Russen von dem deutschen Historiker Grischa Vercamer (2010: 561) als Litauer identifiziert wurden.

${ }^{5}$ Mehr zu dem MTB s. auch Ivoška, 2019b.
} 
lichen Dokumenten des DO, der so viele russische Personennamen sowie damit verbundene Informationen überliefert hat.

\section{Russen im Preußenland um 1399-1409.}

In manchen Dokumenten des DO ggf. vor allem in dem MTB aber auch in den Handfesten des OF 105 und OF $107^{6}$ werden Russen erwähnt, deren Personennamen gebucht sind, ihre Nationalität ist in vielen Fällen mitgenannt. Eben die vertrauliche Nachweisbarkeit der Nationalität von in diesem Beitrag behandelten Personen ist von besonderer Bedeutung für die Erschließung des Einwohneranteils unterschiedlicher Herkunft im Prußenland sowie der Namengebungtendenzen im 14.-15. Jh. Auf den Ordensdokumenten beruhend darf man schlussfolgern, dass es sich sowohl um die im Prußenland ansässigen Russen als auch um kurzfristige Einwanderer bzw. aus Handlungs- oder diplomatischen Gründen in die Dokumente des DO gelangten Namen der Russen handelt.

\section{Ansässige Russen im Prußenland}

Zunächst muss der Gebrauch des Ethnonyms „Russe“ in dem MTB erläutert werden. Die Herausgeber von dem MTB geben alle Variierungen des in den behandelten Dokumenten erwähnten Etnhnonyms an. Grundsätzlich handelt es sich um Formen Ruse, Russe, Russche, und Rusze sowie um die femininen Formen Russekynne, Russkynne, Ruskynne und Russynne.

Die Notierung von unterschiedlichen Formen zur Bezeichnung der Nationalität femininer Formen scheint nicht nur ein Ergebnis der Schreibeigenartigkeit eines gewissen Beamten zu sein. Die frühesten Aufzeichnungen 1399 Russekynne, 1403 Ruskynne regen zu einer Hypothese an. Im altpreußischen Namengut ist ein weiblicher Personenname 1400 Kyne wytwe mit Irem fone (OF $1073^{\mathrm{v}}$ ) bekannt, s. dazu noch Reinhold Trautmann (1925: 45). Ginge man davon aus, dass der Name weder in anderen Ordensdokumenten des Spätmittelalters, noch in späteren vorkommt und von der Tatsache, dass die Angabe der Namen nicht adeliger Frauen in den Ordensdokumenten des behandelten Zeitraumes eine seltene Erscheinung war ${ }^{7}$, so dürfte

\footnotetext{
${ }^{6}$ Blažienè, 2018.

${ }^{7}$ Spräche man von dem OF 105, der eine Menge von Handfesten enthält, die (außer nur manchen datierten von dem 13. Jh.) grundsätzlich aus dem 14. Jh. bis Anfang des 15. Jhs. stammen, würde man feststellen müssen, dass Frauen nur zur Identifizierung der Verwandtschaftsverhältnisse der Männer mitgenannt wurden. Unter ca. 500 Privilegien ist nur eine zu
} 
man vielleicht annehmen, dass kyne auch als ein Appellativum gedient haben mag. Es kann nicht abgelehnt werden, dass es im Altpreußischen Wortschatz *kine 'Weib, Frau' bedeuten konnte, weil man altpreußische Eigennamen auch als Quelle der altpreußischen Lexik erforschen kann (s. Blažienè 2017: 63-85). Demnach wäre es vielleicht erdenkbar, dass zu dem Ethnonym Russe kyne hinzugefügt worden war, um das Geschlecht zu bezeichnen oder eine Russin wurde mit einem apr. PN genannt.

Die variierenden Buchungsformen des Ethnonyms sind mit den Umständen der Führung und der Ausstellung von den Einnahmeund Ausgabedokumenten der Ordensstaatkasse zu verbinden. Die Einträge über die in die Kasse eingegangenen und daraus vergebenen Beträge wurden von etwa einem halben Dutzend von Schreibern, die als Tressler, Ordensschatzmeister oder als deren Hilfskraft fungiert hatten, gemacht, was zu gewissen Schreibunterschiedlichkeiten geführt haben mag.

Die ersten und frühesten Erwähnungen von Russen in dem MTB stammen aus dem Jahre 1399, bei denen es sich zunächst um eine Frau (Russekynne) handelt, für die $10 \mathrm{~m}^{8}$. dem schulzen vom Lybentail ${ }^{9}$ gegeben (MTB 21) wurden, weil er sie aus dem Viehhof genommen hat. Sie wird bald darauf wieder bei der Vergabe von 1 $m$. der selben Russkynne vor eyne ku (MTB 21) erwähnt. Noch eine Russin Namens Kunne ${ }^{10}$, wird im Jahr 1403 zusammen mit ihrem Mann Pauwil erwähnt, weil sie Kunne Pauwil Ruszen wyb zu Danczk am heiligen Leichnam Tag dem Tresslerschreiber Gotschalk 7 Mark Schuld zurückgezahlt hatte (MTB 207). Zu dem PN Kunne vgl. den lit. PN Kùnas, die dt. PN Kuno, Kunis u.Ä. (Gottschald 1954: 390), vgl. noch die poln. PN Kun, Kuna (SSNO III 194).

Eine weitere Zahlung von der 7 Mark großen Schuld wurde von Frau Kunne fünf Jahre später am 29. Juni gemacht, wobei sie nur als dy Pauwel Rüsynne zu Danczk (MTB 451) zu identifizieren ist, ihr

finden, in der eine ehrsame Frau namens Gerdrud / Gertrud erwähnt wurde, die mit ihrem Sohn eine „ewige Lampe einer notdürftigen Kirche“ übergab.

${ }^{8} 10$ Mark.

${ }^{9}$ VTKP (1789: 118, 280) Liebenthal entweder im Landrätlichen Kreis Marienwerder oder auf der Höhe ken Marienburg. S. auch TsPL (1847: 65), Liebenthal — im Regierungsbezirk Danzig, Kreis Marienburg, oder im Regierungsbezirk Marienwerder, Kreis Riesenburg.

${ }^{10}$ Trautmann (1925: 49) gibt die apr. PN Kune, Kuno an, und verbindet ihn mit dem lit. PN Kunas. Er erwähnt aber den Fall aus dem MTB nicht und weist darauf nicht hin, dass mit dem Namen auch Frauen benannt wurden. 
Name wird nicht mitgenannt. Der Name ihres Mannes Pauwel taucht unter den Einträgen des Tresslers auch ein Paar Jahre früher, wo er Pauwel Rusze burger zu Danczik 1401 auch die Schuld von 28 Mark beglichen haben muss (MTB 90). Pauwel mag eine von den Kanzleibeamten dem deutschen (mündlichen und schriftlichen) Namengebrauch adoptierte Namenform von dem russ. Па́вел sein. Hierzu vgl. (Petrovsky 1966: 171-172, Superanskaya: 2005a: 172).

Frau Kunne ist nicht die einzige Russin, die in dem MTB erwähnt wurde. Im Jahr 1405 wurde bei der Auszahlung von $3 \mathrm{~m}$. der frowen Russynne zu Rszecz ${ }^{11}$ (MTB 367) als einer Beihilfe der Name der Begünstigten nicht angegeben. Nicht mehr lässt sich aus den Fällen 1403 eyne Ruskynne zu Marienburg, der 1 firdung ${ }^{12}$ (MTB 244) und 1408 item 18 sch. ${ }^{13}$ den vyemeyden und Ruskynnen zu Ragnit (MTB 488) vergeben wurden, über die beliehenen Frauen erschließen.

Die Ordenskanzleidokumente zeugen davon, dass bei der Auszahlung von Beihilfen oder von Zuschüssen anderer Art sowie bei dem Erhalt von Schulden nur die Namen derjenigen Begünstigten eingetragen wurden, die über gewissen Besitz verfügt hatten und / oder gewisse Dienste dem DO geleistet hatten. Am 16. Juli 1408 zahlte die Ordenskasse unter anderem item $2 \mathrm{~m}$. Supron und Jost syne sone zwen Russen von Pobethen ${ }^{14}$ aus (MTB 496). Supron ist eindeutig ein russ. PN, vgl. den russ. PN Софро́н und Сопро́н (Petrovsky 1966: 203) sowie Софрон von dem akslaw. Софро́ний (Superanskaya: 2005a: 205), wogegen Jost sowohl in der latein- als auch der griechisch-christlichen Namengebungstradition eigen ist. Hierzu vgl. den russ. PN Юcm, Иýcm (Petrovsky 1966: 235, Superanskaya: 2005a: 245) aber auch den Namen, vermutlich, eines Sudauers Jost Sudo von Pobeten dem 1408 item 3 m. gegeben wurden (MTB 490) und dessen Name wahrscheinlich auf den dt. PN Jost, Jöst (GVL 235) zurückzuführen ist. Hierzu vgl. auch den apr. PN Jostaute (OF $105247^{\mathrm{v}}$ ), den lit. Vornamen Jostỹs, der als Abkürzung von dem angegebenen apr. PN interpretiert wird. Jóswird mit lit. jós-iu 'ich werde reiten' verbunden (LVKŽ 209-210). Auch ein Jahr später 1409 wurden noch Wenczken und Symo zwen

\footnotetext{
${ }^{11}$ Nicht ermittelt. Es könnte aber sich, vielleicht, um Rzqdz, $6 \mathrm{~km}$ von Grudziądz handeln (NMP 2015: 132).

${ }^{12} 0,25$ Preußischer Mark.

${ }_{13} 18$ Schilling (ca. 0,3 Preußische Mark).

${ }^{14}$ Pobethen, Kreis Fischhausen, Kirchspiel Pobethen (Blažienè 2000: 119).
} 
Rusen by Nordenburg ${ }^{15} 4$ Mark von dem Tressleramt gegeben (MTB 536). Der Name Wenczken muss eventuell auf dem russ. PN Вячесла́в (Petrovsky 1966: 81) beruhen, dessen Gebrauch sowohl im ost- als auch westslawischen, durch orthodoxen Glauben geprägten Areal weit ausgebreitet war. Die slawisch-orthodoxe Liturgiepraxis kennt auch dem hochgeehrten Heiligen Вячеслав (tschech. Ваилав, dt. Wenzel) gewidmeten Kanon, der ausführlich von Vittorio Springfield Tomelleri (2016) behandelt wurde. Hierzu vgl. noch den poln. PN Więcka, Wiencka (AP VI 177). Der angegebene PN ist eindeutig slawischer Herkunft. Im Fall Symo vgl. die russ. PN Сим, Симео́н und Си́мон (Petrovsky 1966: 201) sowie Си́ма, Си́мон vom latinisierten hebräischen PN Simeon (Superanskaya: 2005b: 202).

In dem MTB werden einige Knechte bzw. Diener der Ordensbeamten erwähnt, deren Namen aber ausgelassen wurden. Im Grunde genommen werden hier Knechte des Bütower ${ }^{16}$ Pflegers gemeint: 1399 die Russe sein knecht, 1399 Ruszen des pflegers knechte zu Butow, 1400 das selbe gelt entfing von uns Rusze des pflegers knecht ${ }^{17}, 1401$ das gelt entfing Rusze syn dyner (MTB 6, 25, $50,95)$.

\section{Annehmbares und Fragliches über die Russen}

Eine komplizierte Frage machen die Nameneinträge aus, die entweder auf russische Herkunft hinweisen oder den Russen eigen gewesen sein durften, können aber auf extralinguistischen Daten beruhend keines Falls eindeutig den Russen zugerechnet werden. Einer von solchen Fällen ist im Jahr 1400 gebuchte Albrecht Rusze burger zu Thorn (MTB 46). Nimmt man Pauwel Rusze burger zu Danczik in Betracht, so ist es wegen des Namens Pauwel glaublich, dass er ein Russe gewesen sein konnte. Es gibt viele Fälle der Zweinamigkeit am Anfang des 15. Jhs. Russe könnte in diesem Fall die Nationalität der Person bezeichnen, während dt. PN Albrecht < Adalbrecht < Adalbert ein alter deutscher männlicher Vorname ist (GVL 50, 41). Ginge es um Buchungen von zweigliedrigen ${ }^{18}$ Personennamen im 16. und späteren Jahrhunderten, dann könnte

\footnotetext{
${ }^{15}$ Nordenburg, Regierungsbezirk Königsberg, Kreis Gerdauen.

${ }^{16}$ Bütow, im Regierungsbezirk Cöslin, Kreis Lauenburg.

${ }^{17}$ Dreimal in der Eintragung erwähnt.

${ }^{18}$ Unter Zweigliedrigkeit werden hier nicht morphologische Bestandteile eines Namens, sondern Benennung einer Person mit zwei Namen verstanden.
} 
man schon von der zweiten Komponente Rusze als einem selbständigen Personennamen sprechen, der sich später neben dem Rufnamen Pauwel bzw. Albrecht zum Nachnamen entwickelte. Die Untersuchungen der Personennamen in dem Prußenland des 13. 15. Jhs. lassen aber nur über die früheste Phase der sich entwickelnden Tendenz, eine Person mit zwei Namen zu benennen, urteilen $^{19}$. Zu behaupten, dass um 1400 ein Russe in Thorn geboren wurde und ihm ein deutscher Vorname gegeben wurde, ist zweifelhaft, trotzdem nicht unmöglich. Die Annahme, dass er wegen irgendwelcher anderen Ursachen zugewandert war, wie das am Ende des 14. Jhs. der Fall mit Litauern gewesen war $^{20}$, ist auch nicht auszuschließen.

Ein nicht leicht lösbares Rätsel gibt auch der zwischen 1401 und 1409 mehr als 11 mal erwähnte Name Ywans von Redemyn ${ }^{21}$ auf, der im Lande zu Dobryn ${ }^{22}$ gewohnt haben musste und mehrmals Auszahlungen von dem DO erhalten sowie Schulden zurückgezahlt hatte. Herr Ywan hatte auch zwei Söhne 1402 her Petrasch hern Ywans son vom Redmyn zu Thorn (MTB 168) und 1409 Niclos von Redmyn her Ywans son (MTB 578), die auch als zahlungspflichtige Personen dokumentiert wurden. Im Jahr 1405 bei der Auszahlung von 4 Marken an her Ywans von Redmyn tochter, wird auch seine Tochter, deren Name nicht angegeben ist, erwähnt. Sie muss zu der Zeit im closter zu Thorn gewohnt haben (MTB 368). Der auf der griechisch-christlichen Namengebungstradition beruhende PN Ywan ist zweifellos russischer Herkunft, hierzu vgl. russ. PN Ива́н (Superanskaya 2005a: 115). Die Namen seiner Söhne, die für beide i.e. latein- und griechisch-christliche Namengebungstraditionen charakteristisch sind, würden gewisse gravierende Fragen erheben, wenn es keine anderen, in demselben MTB dokumentierten Personen Petrasch und Niclos angeführt gäbe, deren Nationalität auch mitgebucht worden ist: 1409 Niclos eyme Russchen felkener der by borgrofe Hannos von Norenberg ${ }^{23}$ was (MTB 594) und 1408 Petersch dem Russchen felkener als her weder ken Littawen zoch

${ }^{19}$ Ivoška (2018: 37, 56-57).

${ }^{20}$ Ivoška (2018: 38-39, 185).

${ }^{21}$ Radomin, Dorf in der Gemeinde Nidzica, $6 \mathrm{~km}$ südlich von Nidzica (NMP 2015: 41).

${ }^{22}$ Döbringen oder Alt- und Neu Döbrin, Kreis Mohrungen (Goldbeck 1785: 36).

${ }^{23}$ Nörenberg, im Regierungsberzirk Stettin, Kreis Saazig. 
(MTB 507) sowie 1408 Peter dem Russchen felkener zu Schoken ${ }^{24}$ zusammen mit zwei anderen nicht identifizierbaren Ruschen felkenern, den $1 \mathrm{~m}$. und item 14 scot vor 2 par stefelen und vor 2 par schu (MTB 487) in demselben Jahr vergeben wurden. Zu der russischen Entsprechungen von dem PN Niclos s. russ. PN Никола́ŭ (Petrovsky 1966: 166, Superanskaya 2005a: 165). Der russ. PN Petrasch beruht wahrscheinlich auf dem russ. PN Пёmp, der zahlreiche Variierungen von russ. PN aufweist. Hierzu vgl. den russ. PN Петра́u (Superanskaya: 2005a: 204) und dessen Suffigierenden Петрашко́в, Петрашо́в (Ganzhina 2000: 365).

Die Tatsache, dass in keinem von den 11 Erwähnungsfällen von Ywan und seiner Familie seine Nationalität erwähnt wird und dass sich seine Tochter in Thorn im Kloster befand, bringt gewisse Verwirrung. Sie müssen eine von den völlig assimilierten Familien gewesen sein, anders ist nicht überzeugend, dass ein aus dem heimatlichen Russland zugewanderte, wahrscheinlich christlichorthodoxen Glauben praktizierende Russe seine Tochter ins christlich-katholische Kloster gehen lassen hätte.

Zwischen 1400 und 1409 werden auch andere Einwohner namens Ywan genannt und zwar 1400 Ywan von Grotkow ${ }^{25}$ (MTB 46), der eine dem DO einen nicht genannten Betrag an die Ordenskasse bezahlt hatte, 1408 Ywan des meysters jungen (MTB 491), und 1409 Ywan von Cleyne Belkaw ${ }^{26}$ im Danker gebyte (MTB 563), dem $4 \mathrm{~m}$. gegeben wurden und 1408 Ywan Truchen son von Pobeten (MTB 490), dem $1 \mathrm{~m}$. gegeben wurde. Der letzte Fall ist sehr aussagekräftig, er macht einen von wenigen Fällen, wo die Nationalität des Beliehenen nicht explizit angegeben ist, die Namen des Vaters und des Sohnes sind aber beide russischer Herkunft vgl. russ. PN Иван und russ. PN Тру́ха́н (Superanskaya: 2005b: 126, 247). Es seien auch die russ. PN Труха, Трухан, Трухин bemerkt, die alle im 15.-16. Jh. zu Nowgorod (russ. Новгород) erwähnt wurden (Veselovsky 1974: 323). Kurze Anmerkungen über enge geopolitische und Handelsverhältnisse zwischen dem DO, dem Großfürstentum Litauen und der russischen Kaufmannsstadt Nowgorod sind unter allem auch in dem MTB (360, 474)

\footnotetext{
${ }^{24}$ Schaaken, Kreis Königsberg, Kirchspiel Schaaken (Blažienè 2000: 141).

${ }^{25}$ Nicht ermittelt. Es mag sich, vielleicht, um Grodkowo, in der Gemeinde Sierpc, 6 km südöstlich von Sierpc handeln (MTB 1999. 377).

${ }^{26}$ Mag vielleicht Biatkowo, in der Gemeinde Golub-Dobrzyń, $3 \mathrm{~km}$ südöstlich von Golub-Dobrzyń (NMP 1996) 148.
} 
aufgezeichnet ${ }^{27}$. Daraufhin darf behauptet werden, dass die Familie wirklich russisch gewesen sein musste. $\mathrm{Zu}$ erwähnen ist hier auch ein Litauer namens Ywan, der 1404 zusammen mit 12 anderen litauischen Gesinden zur Losung gestellt wurde ${ }^{28}$ (MTB 315). Es gibt nur eine Erwähnung von 21 Ywan-Erwähnungen, wo die Nationalität mitgenannt wurde: 1409 Ywan und Jorgen zwen Russen von der Pruschenylaw ${ }^{29}$ (MTB 531), denen $4 \mathrm{~m}$. ausgezahlt wurden. Die Notierung des Namens des zweiten Begünstigten muss verdeutscht sein und auf den russ. Юрий, Георгий und dessen nowgorodisch-dialektale Form Гюргий, die ihrerseits dem lit. PN Jurgis entspricht, zurückgeführt werden (Zaliznyak 2004: 104).

Es gibt noch einen Eintrag in dem Beliehenenregister und zwar über 1404 Ywan Perbans Sohn, der zur Splitter ${ }^{30}$ gesetzt und im eyn wyb vor $2 \mathrm{~m}$. gekauft wurde (MTB 318). Der Name von Ywans Vater Perbans macht einen Sonderfall aus. Es kann auf die russ. PN Первыи (Veselovsky 1974: 241) und Первой (Kazansky 1995: 165) zurückgeführt werden, den das Kardinalzahlwort russ. nервыц, $(-я)$ 'der, die erste' zugrunde liegt. Sich auf die Variabilität der suffigierten Personennamen stützend ${ }^{31}$ darf man vielleicht annehmen, dass es auch einen russ. PN *Перван (*Pervan) gegeben haben mag. Die auf Zahlwort basierende Namenbildungstradition ist in vielen Sprachen bekannt und war besonders in Latein, Altindischen, Russischen und Litauischen produktiv gewesen (Kazansky 1995: 164-165). Die Verwechslung von den ähnlich lautenden labialen Konsonanten $b / w$ bzw. $\sigma / \beta$ kann bei der Notierung der fremden, ungewöhnlichen Personennamen durch Beamten des DO aufgetreten sein. Hierzu vgl. auch einen analogischen Fall, den der apr. PN Narwoto (OF $105242^{r}$ ) bildet und dessen Form im prußischen bzw. litauischen Personennamengut nicht nachgewiesen ist. Die Form Narbut- ist dagegen unter den litauischen Personennamen weit verbreitet ${ }^{32}$. Die in einigen Ordensdokumenten gebuchte apr. PN Perbande (Trautmann 76)

\footnotetext{
${ }^{27}$ Die Frage der eventuell aus Nowgorod stammenden und im Preußenland ansässigen Russen hat Blažienè (2018: 121) bei der Deutung des russ. PN Carpe angegangen.

${ }^{28}$ Ivoška (2019: 99, 103-104).

${ }^{29}$ Preußisch Eylau, Kreis Pr. Eylau (Blažienė 2005: 159).

${ }^{30}$ Splitter, Alt Splitter, Kreis Insterburg, Lit. Spitré, (MLE IV 2009: 521).

${ }^{31}$ Hierzu vgl. auch den russ. PN Первуша (Veselovsky 1974: 241).

${ }^{32}$ Mehr dazu s. Ivoška (2018: 95).
} 
muss mutmaßlich mit dem Namen von Ywans Vater nicht viel zu tun haben.

Summierend muss hervorgehoben werden, dass es bei den 21 Erwähnungen von dem PN Ywan um 8 Personen handelt, von denen nur Ywan Bruder von Jorgen von den Ordensbeamten als Russe bezeichnet wird. Andere 7 Fälle bleiben direkt nicht identifizierbar, auf Grund der kontextgebundenen Daten lassen sich aber bestimmte Vermutungen äußern: spräche man zunächst von dem Ywan von Redemyn, der zwei Söhne hatte, die mit eventuell russischen Namen (die in dem MTB auf verdeutschte Gebrauchsweise Petrasch und Niclos gebucht sind), könnte man, vielleicht behaupten, dass sie Russen gewesen sind, wie auch in dem Fall von Ywan Truchen Sohn von Pobethen. Ywans Tochterfrage, wird aber durch die Auslassung deren Namens und durch die Angaben über ihr Beisein im Thorner Kloster in den finanziellen Dokumenten des DO, zu einem komplizierten Kasus, der eine verlässliche Annahme über die Nationalität der ganzen Familie erschwert. Die Einzelerwähnungen von andren Personen Ywan genannt - Ywan von Cleyne Belkaw, Ywan von Grotkow, Ywan Meisters Sohn u.a. - liefern keine Andeutungen, die mindestens vermutlich über ihre Nationalität zu urteilen erlauben würden.

\section{Russisch-litauische Diener und Herzöge}

Unter den Russen, die Siedler des Prußenlandes waren, werden, wie bereits hingewiesen, auch diejenigen dokumentiert, die entweder nach Prußenland dienstlich gesandt wurden und dort gewisse Zeit verbracht haben mussten, oder in bestimmten diplomatischen Beziehungen zu dem DO waren. Von dem DO erhielten Zahlungen die Falkenzüchter und -lieferer des litauischen Herzogs Wytowt. Unter Einträgen über Ausgaben der Ordenskasse sind auch die über die Zahlungen von 2 1/2 schok behm. gr. ${ }^{33} 1408$ an Petresch Herzoge Wytawten felkener, der dem Hochmeister der DO Falken und Habichte geliefert hatte (MTB 478), zu finden. Es mag sein, dass derselbe Falkenzüchter ein paar Monate später wieder erwähnt wurde, indem Petresch dem Ruschen felkener am dinstage noch visit. Marie 1 Mark bezahlt wurde (MTB 491). Dieses Mal ist er als Russe identifiziert. Es gibt noch einen Ruesse, den Falkner von Herzog Wytowt namens Sum, der am 15. März 1408 die falken brochte von Wytowten (MTB 471) und die Herkunft dessen Namens

${ }^{33}$ Ein Schock böhmische Groschen ist 1 Mark 12 Skot gleich. 
nicht klar ist. Er mag, vielleiht, als eine verkürzte Form des russ. akslw. PN Сумео́н gelten (Superanskaya: 2005b: 229). Unter den im MTB erwähnten Russen ist auch kurz auf 1400 zwen jungen herzogen S. 83, den zwen jungen herzogen von Ruszen S. 86 und 1401 item $1 \mathrm{~m}$. Hannus Surwillen des meisters dyner zerunge, als her dy Russchen Herzoge usrichte S. 116 zwei russischen Herzoge hingewiesen, deren Namen leider ausgelassen sind.

\section{Fazit}

Nach der Untersuchung der zugrunde liegenden, die Sozialverhältnisse im mittelalterlichen Prußenland betreffenden Werke und etlicher historischer Dokumentensammlungen des DO muss bemerkt werden, dass es eine gewisse Lücke in der historischwissenschaftlichen Kenntnis geben mag. Diese Lücke entsteht zwischen den Tatsachen, die in den wichtigsten Geschichtsquellen durch Dokumente belegt sind, und den im wissenschaftlichen Umsatz diverser Forschungsrichtungen vorhandenen Fachkenntnissen. Würde man eine Frage stellen, was man über die Bewohner russischer Herkunft in dem vorherzoglichen Prußenland weiß, wäre es wahrscheinlich kompliziert, sie ohne weiteres $\mathrm{zu}$ beantworten. Die Veröffentlichungen, in denen die behandelte Problematik sparsam angesprochen wird, sind gering und genaue Daten über die Abstammung der Russen, deren Anzahl in dem betroffenen Areal sowie ihr Stand in der Gesellschaft fehlen. Die Annahmen, dass die Aufmerksamkeit der Forscher auf alle ethnischen Minderheiten, die keine wesentliche Rolle in den sozialen, wirtschaftlichen und kulturellen Vorgängen gespielt hatten, kaum gelenkt worden ist, regen zur notwendigen Minderung des Mangels an zusammenhängenden Forschungen an. Die historischen Geschichtsquellen und zahlreichen Archivbestände scheinen eine gute Basis dafür schaffen zu können.

Ein größerer Teil der Russen bzw. Personen, die Namen russischer Herkunft getragen hatten und in der Hauptquelle des vorliegenden Beitrages erwähnt wurden, haben sowohl in West(Danziger Gebiet, Marienwerder und Gebiet Thorn) als auch in Ostpreußen (Kreis Fischhausen, Gerdauen, Osterode, Preußisch Eylau) gewohnt. Andere hatten das Prußenland aus dienstlichen Gründen besucht, müssen aber von nicht lokalisierbaren Orten gestammt haben. 


\section{Literatur}

AP - Antroponimia Polski od XVI do końca XVIII wieku. T. VI: V-Ż pod redakcją Aleksandry Cieślikowej przy współpracy Halszki Górny i Katarzyny Skowronek. Kraków, 2016.

Biskup, M. 1991: Das Problem der ethnischen Zugehörigkeit im mittelalterlichen Landesbau in Preußen. In: Jahrbuch für die Geschichte Mittel- und Ostdeutschlands 40. Berlin: Colloquium Verlag, 4-25.

Biskup, M.1991: Etniczno-demograficzne przemiany Prus krzyżackich w rozwoju osadnictwa w średniowieczu (o tak zwanym nowym plemieniu Prusaków). In: Kwartalnik historyczny. Rocznik XCVIII Nr. 2. Warszawa: Polska Akademia Nauk Instytut Historii, 45-67.

Blažienè, G. 2000: Die baltischen Ortsnamen im Samland (=Hydronymia Europaea, Sonderband II), Stuttgart: Franz Steiner Verlag.

Blažienè, G. 2005: Baltische Ortsnamen in Ostpreußen (=Hydronymia Europaea, Sonderband III), Stuttgart: Franz Steiner Verlag.

Blažienè, G. 2017: Altpreußische Eigennamen als Quelle der altpreußischen Lexik. In: Incontri Baltistici in Pisa. Studi e saggi / a cura di Petro U. Dini. Novi Ligure: Edizione Joker, 63-85.

Blažienè, G. 2018: Zu den russischen Personennamen in den Folianten des Deutschen Ordens. Indo-European Linguistics and Classical Philology 22, 109-124.

Erlen, P. 1992: Europäischer Landesausbau und mittelalterliche deutsche Ortsiedlung. Ein struktureller Vergleich zwischen Südfrankreich, den Niederlanden und dem Ordensland Preußen. Historische und Landeskundliche Ostmitteleuropa-Studien 9. Marburg an der Lahn: Johann-Gottfried-Herder-Institut.

Forstreuter, K. 1937: Buchbesprechung: Kasiske, Karl: Die Siedlungstätigkeit des Deutschen Ordens im östlichen Preußen bis zum Jahre 1410hung. In: Jahrbücher zur Geschichte Osteuropas. Hrsg. von Hans Uebersberger Berlin, Jahrgang 2. Berlin: Verlag Priebatschs Buchhandlung Breslau, 501.

Ganzhina, I. M. 2000: [Dictionary of modern Russian surnames]. Moscow: AST, Astrel'.

Ганжина, И. М. 2000: Словарь современных русских фамилий. М.: АСТ, Астрель.

Goldbeck, J. F. 1789: Volständige Topographie des Königreichs Preußen. Zweiter Theil welcher die Topographie von West-Preußen enthält. Marienwerder: Gedruckt in der Königl. West-Preuß. Kanterschen Hofbuchdruckerei.

Gottschald, M.1954: Deutsche Namenkunde. Berlin: Verlag Walter de Gruyter u. Co.

GVL - Duden. Das große Vornamenlexikon. Bearbeitet von Rosa und Volker Kohlheim. Berlin, Mannheim, Zürich: Dudenverlag, 2014.

Ivoška, D. 2019a: Widerspiegelung der prußischen Kultur in den Grenzbeschreibungen des Deutschen Ordens. In: Preussenland 10. 
Jahrbuch der Historischen Kommission für ost- und westpreußische Landesforschung und der Copernicus-Vereinigung für Geschichte und Landeskunde Westpreußens, 7-23.

Ivoška, D. 2019b: Litauische historischen Personennamen in dem „Marienburger Tresslerbuch der Jahre 1399-1409“. In: Acta Linguistica Lithuanica 81, 97-109.

Ivoška, D. Baltische Eigennamen in den Dokumenten des Deutschen Ordens. Dissertation zur Erlangung der Doktorwürde. Vilnius: Lietuviu kalbos institutas.

Janeczek, A. 1987: Exceptis schismaticis: Upośledzenie Rusinów w przywilejach prawa niemieckiego Władysława Jagiełły. In: Przeglad Historyczny 75/3, 527-542.

Kasiske, K. 1934: Die Siedlungstätigkeit des Deutschen Ordens im östlichen Preußen bis zum Jahre 1410. Königsberg i. Pr.: Kommissionsverlag Gräfe und Unzer.

Kazansky, N. N. 1995: Indo-European Onomastics as an Historical Source. In: The Journal of Indo-European Studies 23, 157-177.

Kowalska-Urbankowa, Z. 1987: Unia Polski i Litwy w latach 1385-1413 w najnowszej historiografii polskiej. In: Analecta Cracoviensia: (studia philosophico-theologica edita a professoribus Cracoviae) XIX, 207-221.

LPDB - Lietuviu pavardžiu duomenu bazè. Zutritt http://lkiis.lki.lt

LVKŽ - Kuzavinis, K., Savukynas, B. Lietuviu vardu kilmès žodynas. Vilnius: Mokslas, 1987.

Małłek, J. 2003: Migracje ludności niemieckiej, polskiej i litewskiej na ziemie pruskie w XIII-XVIII wieku. In: Komunikaty MazurskoWarmińskie 4, 431-441.

MLE - Mažosios Lietuvos enciklopedija, 4 Rahn-Žvižežeris. Vilnius: Mažosios Lietuvos fondas, Mokslo ir enciklopedijų leidybos institutas, 2009.

Mortensen, G. 1927: Beiträge zu den Nationalitäten- und Siedlungsverhältnissen von Pr. Litauen. Memel: Memelland-Verlag, BerlinNowawes.

MTB - Das Marienburger Tresslerbuch der Jahre 1399-1409. Hrsg. von Archivrath Dr. Joachim, Königsberg In Preußen: Verlag von Thomas \& Oppermann, 1898.

NMP I - Nazwy miejscowe polski. Historia, Pochodzenie, Zmiany, Bd. I A-B (pod redakcja Kazimierza Rymuta, Barbary CzopekKopciuch), 1996, Kraków: Instytut Języka Polskiego.

NMP III - Nazwy miejscowe polski. Historia, Pochodzenie, Zmiany, Bd. III E-I (pod redakcją Kazimierza Rymuta, Barbary CzopekKopciuch), 1999, Kraków: Instytut Języka Polskiego.

NMP X - Nazwy miejscowe polski. Historia, Pochodzenie, Zmiany, Bd. X Ra-Re (pod redakcja Kazimierza Rymuta, Barbary CzopekKopciuch), 2015, Kraków: Instytut Języka Polskiego. 
OF - Ordensfolianten aus dem Geheimen Staatsarchiv „Preußischer Kulturbesitz".

Petrovsky, N. A. 1966: [Dictionary of Russian Personal Names]. M.: Sovetskaya entsiklopediya. Петровский, Н. А. 1966: Словарь русских личных имен. М.: Советская энциклопедия.

SSNO III - Stownik staropolskich nazw osobowych. Wrocław-Warszawa; Kraków; Gdańsk: Zakład Narodowy imienia Ossolińskich, Wydawnictwo Polskiej Akademii Nauk, 1965.

Superanskaya, A. V. 2005a: [Dictionary of Russian Names]. Moscow. Суперанская, А. В. 2005а: Словарь русских имён. М.: Эксмо.

Superanskaya, A. V. 2005b: [Modern dictionary of personal names. Comparison, origin, spelling]. Moscow: Airis press.

Суперанская, А. В. 2005b: Современный словарь личных имён, сравнение, происхождение, написание . М.: Айрис пресс.

Tomelleri, V. S. 2016: Zu den Theotokia im Wenzelskanon / Богородичны в каноне Св. Бячеславу. In: Slověne 1, 7-69.

Trautmann, R. 1925. Die altpreußischen Personennamen. Göttingen: Vandenhoeck \& Ruprecht.

TsPL - Topographisch-statistisches Post-Lexicon oder Alphabetisches Verzeichnis sämtlicher in Deutschland und der Schweiz gelegenen Städte, Marktflicken und namhaften Dörfer mit besonderer Bezeichnung der Postnastalten, Eisenbahnstationen, Fabrikorte und Handelsplätze. Zum Gebrauche für Postanstalten, Expeditionen, Bureaux und Comptoires. Hrsg. von Hermann Martens. Grimma: Verlag von J. M. Gebhartdt, 1847.

Vercamer, G. 2010: Siedlungs-, Sozial- und Verwaltungsgeschichte der Komturei Königsberg in Preußen (13. -16. Jahrhundert). Marburg: N.G. Elwert Verlag.

Veselovsky, S. B. 1974: [Onomasticon. Old Russian Names, Nicknames and Surnames]. Moscow: Nauka.

Веселовский, С. Б. 1974: Ономастикон. Древнерусские имена, прозвища и фамилии. М.: Наука.

Wenskus, R. 1975: Der deutsche Orden und die nichtdeutsche Bevölkerung des Preußenlandes mit besonderer Berücksichtigung der Siedlung. In: Konstanzer Arbeitskreis für Mittelalterliche Geschichte. Vorträge und Forschungen: Die deutsche Ostsiedlung des Mittelalters als Problem der europäischen Geschichte 18, 417-438.

Zaliznyak, A. A. 2004: [Old Novgorod-Dialect. $\left(2^{\text {nd }}\right.$ edition, based on new found materials in 1995-2003)]. Moscow: Yazyki slavyanskoy kul'tury. Зализняк, А. А. 2004: Древненовгородский диалект. (2е издание, переработанное с учетом материала находок 19952003 гг.). М.: Языки славянской культуры. 